\title{
Postural Synergies and their Development
}

\author{
Mark L. Latash, ${ }^{1}$ Vijaya Krishnamoorthy, ${ }^{2}$ John P. Scholz ${ }^{2}$ and Vladimir M. Zatsiorsky ${ }^{1}$ \\ ${ }^{1}$ The Pennsylvania State University, University Park, PA 16802; \\ ${ }^{2}$ The University of Delaware, Newark, DE 19716, USA
}

\begin{abstract}
SUMMARY
The recent developments of a particular approach to analyzing motor synergies based on the principle of motor abundance has allowed a quantitative assessment of multieffector coordination in motor tasks involving anticipatory adjustments to self-triggered postural perturbations and in voluntary postural sway. This approach, the uncontrolled manifold (UCM) hypothesis, is based on an assumption that the central nervous system organizes covariation of elemental variables to stabilize important performance variables in a taskspecific manner. In particular, this approach has been used to demonstrate and to assess the emergence of synergies and their modification with motor practice in typical persons and persons with Down syndrome. The framework of the UCM hypothesis allows the formulation of testable hypotheses with respect to developing postural synergies in typically and atypically developing persons.
\end{abstract}

\section{KEYWORDS}

synergy, posture, practice, variability, Down syndrome

Reprint requests to: Mark Latash, Rec.Hall-266N, Dept of Kinesiology, The Pennsylvania State University, University Park, PA 16802, USA. e-mail: mll11@psu.edu

\section{INTRODUCTION}

Bipedal vertical posture is inherently unstable because of the anatomy of the human body, which can be viewed as an inverted pendulum with a rather high center of mass standing on a rather small support. Several factors can be viewed as complicating the task of maintaining vertical posture. First, there are several joints along the axis of the body. Apparently, muscle action at the joints has to be coordinated to keep the projection of the center of mass within the area of support. Second, there are frequent changes in external conditions, mechanical and sensory, that may be seen as perturbations for the posture. Apparently, muscles have to be able to correct mechanical effects of such perturbations to avoid losing balance. Third, voluntary motor actions by standing persons by themselves can be sources of postural perturbations as well, due to the action of inertial and coupling forces and to changes in the relative position of body segments leading to changes in the location of the center of mass of the body.

Postural studies have typically addressed these three components of the task of standing (or sitting, or maintaining any other posture) as reflected in the following three phenomena, postural sway (reviewed in Winter et al., 1996), short-latency postural responses (reviewed in Nashner et al., 1989), and anticipatory postural adjustments (APAs, reviewed in Massion, 1992). Studies of postural development have also addressed these three phenomena. In particular, children show increased postural sway (Woollacott \& Burtner, 1996. Van der Fits et al., 1999) - the 
emergence of short-latency postural adjustmentsseveral months after acquiring the ability to stand upright (Haas et al. 1986. Woollacott et al., 1998. Wollacott \& Assaiante, 2002), with an even longer delay before typical APAs emerge (van der Fits et al., 1999. van der Heide et al., 2003). Many of these studies invoked the notion of postural synergies. yet, typically, this notion is used rather loosely, without a clear operational definition that would allow, for example, quantifying "strength" of a synergy and, in particular to distinguish a synergy from a "non-synergy". The main purpose of this paper is to review a certain approach to motor synergies that allows their quantification and can be used, in particular, to track changes in postural synergies with typical and atypical development. We would like to start, however, with a brief historical introduction.

\section{ELEMENTS OF HISTORY}

No agreement has been reached on what a postural synergy is. Moreover, no agreement has been reached on what any synergy is. The word itself means "work together", but this definition is obviously too general to be used in quantitative research. Many studies have implied under this word patterns of kinematic, kinetic, or electromyographic (EMG) variables that are reproduced or scaled in parallel over the time course of an action or across repetitive trials. For example, in a recent paper, D'Avilla and co-authors (2003) defined muscle synergies as coherent activations, in space or time, of a group of muscles.

This understanding of motor synergies can be traced back to the great neurologists of the end of the $19^{\text {th }}$ century, Hughlings Jackson (1889) and Babinski (1899). Nevertheless, for most contemporary scientists, the notion of synergy is associated with the name of Bernstein, who introduced a multi-level theory of control of movements, in which one level was termed the level of synergies
(Bernstein, 1947, 1967). At this level, coordinated actions by sets of effectors were supposed to emerge. Most contemporary studies use the word "synergy" in a Bernsteinian sense.

With respect to postural synergies, examples from recent studies include the ankle and the hip strategies (Horak \& Nashner, 1986), a multi-link strategy and muscle amplitude synergies (Allum et al., 1989. Allum \& Honneger, 1993), the ankle, knee, and hip eigenmovements (Alexandrov et al., 1998), axial synergies (Crenna et al., 1987), and reciprocal and co-contraction strategies (Slijper \& Latash, 2000). All these examples are based on a common assumption that a regularity in the behavior of a set of elements is a sufficient sign to claim an existence of a synergy. We would like to suggest, however, an alternative approach to quantitative analysis of synergies that follows the traditions of Gel fand and Tsetlin (1966).

\section{THE PROBLEM OF MOTOR REDUNDANCY AND THE PRINCIPLE OF ABUNDANCE}

The notion of synergy is closely linked to the problem of motor redundancy: How does the central nervous system make choices from a seemingly infinite number of possibilities typical of most everyday motor tasks and all levels of analysis of movement production? For example, imagine a pointing task that requires a certain trajectory of a finger tip. How to select a combination of joint angles to produce the required trajectory? How to select muscle forces that would implement the joint rotations? How to select patterns of firing of individual motor units that would produce required muscle forces? And so on. All these problems are characterized by a common feature: They involve fewer constraints than elements (Turvey, 1990. Latash, 1996). Bernstein (1947) viewed synergies as patterns of joint rotations, muscle activation patterns, etc. that are learned with practice and used to find 
particular solutions for the problems of motor redundancy. According to his commonly cited formulation, synergies help eliminate redundant degrees-of-freedom.

This predominant opinion has been challenged only recently with an alternative view expressed as a principle of abundance (Gelfand \& Latash, 1998, 2002; see also Gelfand \& Tsetlin, 1966). According to this opinion, degrees-of-freedom are never eliminated but always used to minimize errors in functionally important performance variables. The controller organizes all the elements such that they compensate for each others spontaneous errors as well as for potential errors that may result from unpredictable forces acting from the environment. In some studies, this aspect of the functioning of synergies has been addressed as "error compensation" (Latash et al., 1998).

According to the principle of abundance, synergies may be defined as co-varying changes in the outputs of individual elements of a multielement system (addressed further as elemental variables, EVs) that stabilize a value or a time profile of an important performance variable produced by the system. As shown in the next subsection, this definition makes it possible to quantify synergies with respect to the stabilization of different performance variables. In other words, it allows asking a multi-element system a question: Are you a synergy with respect to this particular performance variable?

\section{GOOD AND BAD VARIABILITY AND THE UNCONTROLLED MANIFOLD HYPOTHESIS}

Variability is present in all natural motor actions. Recently, the attitude toward motor variability has shown a shift from considering it as a sign of imperfection of the neuromotor system, a "noise", to viewing it as an important component of the functioning of the apparatus for the production of movements (reviewed in Newell \&
Corcos, 1996). In multi-element systems, motor variability can be analyzed at the level of EVs and at the level of important performance variables. For example, during force production tasks by a single element, e.g., a digit or a joint, variability in the force output quantified as force standard deviation increases nearly linearly with the total level of force (Newell et al., 1984). However, when more than one element contributes to the total force output, for example when several digits press in parallel to produce a certain level of the total force, this relation breaks down, and total force variability shows a minimal dependence on the force level (Latash et al., 2001. Shinohara et al., 2003). This observation suggests that variations in the force outputs of individual digits are not independent, but rather co-vary to keep the total force variability from increasing with an increase in the total force.

Figure 1 illustrates how this variability can happen for a task of a constant total force production by two fingers pressing in parallel. The ellipses in the figure show the hypothetical distributions of data points over a set of trials at producing the total force of $5 \mathrm{~N}, 10 \mathrm{~N}$, and $20 \mathrm{~N}$. Note that the range of forces produced by each finger increases with the total force (cf. Newell \& Carlton, 1988). However, this variability has two components. One is aligned along lines $\mathrm{F}_{1}+\mathrm{F}_{2}=\mathrm{F}_{\text {TARGET}}$; this variability does not affect the total force. The other component is orthogonal to these lines and leads to changes in the total force. In other words, the former variability is 'good' in a sense that it keeps the important performance variable, the total force, at a desired level. The latter variability is 'bad' in a sense that it leads to changes in the total force. Note that an increase in the total force leads to changes primarily in the 'good' variability, thus keeping the variability of the total force virtually unchanged. Note that the terms 'good' and 'bad' relate to the effects of variability in the space of elements on the variability of a particular performance variable. 


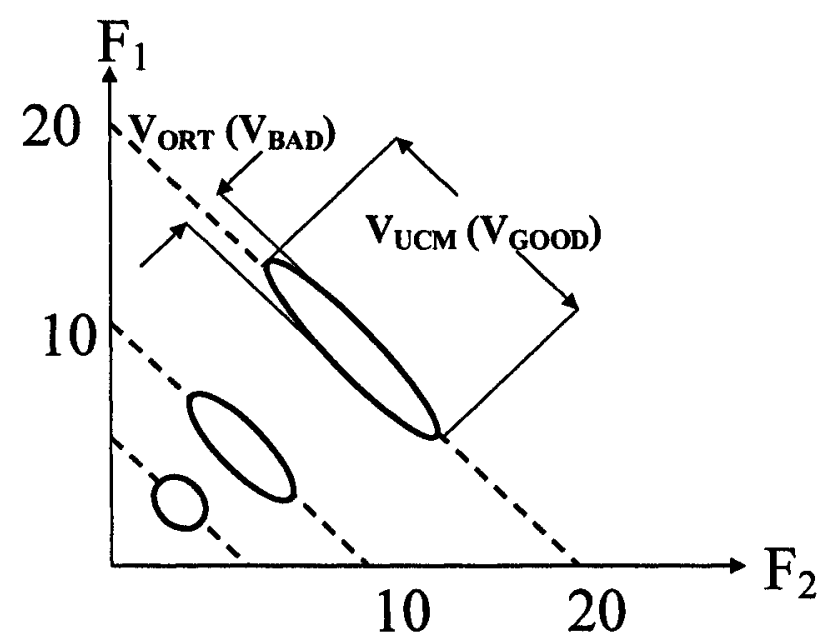

Fig. 1: Hypothetical distribution of data points across many trials when the person is asked to produce a certain level of the total force by pressing on force sensors with two effectors. The three dashed lines correspond to the total forces of $5 \mathrm{~N}, 10 \mathrm{~N}$, and $20 \mathrm{~N}$. They represent uncontrolled manifolds (UCMs) for these total force levels. The natural variability of the forces of the effectors $\left(F_{1}\right.$ and $\left.F_{2}\right)$ increases with the total force. However, this is accompanied by no changes in the variance of the total force $\left(\mathrm{V}_{\mathrm{ORT}}\right.$ or $\left.\mathrm{V}_{\mathrm{BAD}}\right)$ with an increase in variance that does not affect total force $\left(\mathrm{V}_{\mathrm{UCM}}\right.$ or $\left.\mathrm{V}_{\mathrm{GOOD}}\right)$.

During any action, variability at a particular level of description of the neuromotor system can be represented as a combination of 'good' and 'bad' components with respect to different performance variables.

The illustration in Fig. 1, which is a rather accurate reflection of findings in experiments with multi-finger force production (Latash et al., 2001, 2003b), suggests that the controller organizes covariation of the EVs in such a way that their variability is primarily restricted to a sub-space in the space of EVs. This sub-space has been termed an "uncontrolled manifold", UCM.

Correspondingly, a UCM hypothesis suggests that a controller organizes outputs of elements within a multi-element system in such a way that EVs are mostly restricted to the UCM (Schöner, 1995). This organization does not eliminate any degrees-of-freedom but rather uses them to limit the amount of 'bad' variability with respect to important performance variables or several performance variables.

\section{ANALYSIS WITHIN THE UCM HYPOTHESIS}

The UCM hypothesis can be applied to the analysis of various motor actions using different sets of EVs. Such analysis involves several important steps.

First, obviously a task and a level of analysis have to be selected. The UCM hypothesis has been used to analyze a variety of tasks including multijoint pointing, multi-finger force production, standing, etc. (reviewed in Latash et al., 2002a, 2003b). The analyses were performed using kinetic 
kinematic, and electromyographic (EMG) variables. However, recording the output variables of apparent elements involved in a task does not mean, by itself, that these may be viewed as EVs. EVs should be at least hypothetically independent from each other in the absence of task-specific control. This may not be true for at least some of the mentioned output variables.

Second, a set of EVs has to be defined. During kinematic analysis, EVs have been commonly associated with rotations in individual joints. This is a non-trivial assumption because of the joint coupling, in particular by bi- and multi-articular muscles. During the analysis of finger forces, the phenomenon of force production by explicitly noninvolved fingers (enslaving, Zatsiorsky et al., 1998, 2000) does not allow viewing individual finger forces as EVs. Hence, a different set of variables termed force modes has been introduced (Latash et al., 2001. Danion et al., 2003) and used for analysis within the UCM hypothesis. Since Hughlings Jackson (1889), researchers did not believe that individual muscles are controlled independently by the brain. Hence, UCM analysis of EMG variables had to start with defining the appropriate EVs (muscle modes, Krishnamoorthy et al., 2003a). Muscle modes introduced in the mentioned studies corresponded to parallel scaling of the activity of sets of muscles.

Third, a control hypothesis has to be formulated, i.e. a hypothesis on a performance variable that is supposedly stabilized by co-varied changes of EVs. Setting a motor task implies a particular performance variable-for example, asking a person to produce a time profile of the total force by a set of effectors implies the stabilization of the total force. However, this step is also non-trivial: In several studies, subjects have been shown to stabilize not the variable explicitly emphasized in the task formulation and reflected in the provided visual feedback but another performance variable (Scholz et al., 2002. Latash et al., 2002c).

On the other hand, several variables can be stabilized to different degrees at the same time if the number of EVs is large enough (Latash et al., 2001. Kang et al., 2004). Motor tasks are sometimes ambiguous with respect to variables that have to be stabilized (controlled). For example, the task of quiet standing can be viewed as associated with the stabilization of the location of the center of mass or the location of the resultant force acting on the body (center of pressure, COP) or visual perception. One of the strengths of the UCM hypothesis is that it allows testing different control hypotheses based on the same data set and producing quantitative indices of stabilization of different performance variables.

Fourth, a Jacobian matrix ( $\mathbf{J})$ of the system has to be computed, i.e. a matrix that reflects effect of small changes in EVs on changes in a selected performance variable. In some cases, $\mathbf{J}$ can be explicitly computed, for example in kinematic studies of multi-joint pointing (Scholz \& Schöner, 1999. Scholz et al., 2000. Domkin et al., 2003). In other cases, $\mathbf{J}$ has to be discovered using experimental techniques (Latash et al., 2001. Krishnamoorthy et al., 2003a,b).

Fifth, the null-space of the $\mathbf{J}$ matrix has to be computed corresponding to a particular value of the selected performance variable. Commonly, an average value of the performance variable, computed across trials, has been used at this step. The null-space is the UCM for this performance variable. It represents a linear estimate of the subspace in the space of elemental variables containing all possible combinations of EVs that lead to the same value of the performance variable.

Sixth, finally, an index of variability (commonly, the variance) in the EV space across trials or, in some special cases, along a trial (Scholz et al., 2003) has to be quantified within the UCM and in a sub-space orthogonal to the UCM per dimenision within each of these two sub-spaces. If the variance within the UCM ( $\left.V_{U C M}\right)$ is greater than the variance orthogonal to the UCM ( $\left.V_{\text {ORT }}\right)$, then a conclusion can be drawn that this particular 
performance variable is indeed stabilized by a covariation of EVs, i.e. there is an appropriate synergy. Different indices, including the ratio $\mathrm{V}_{\mathrm{UCM}} / \mathrm{V}_{\mathrm{ORT}}$ and the normalized difference between $\mathrm{V}_{U C M}$ and $\mathrm{V}_{\mathrm{ORT}}$, have been used to quantify such synergies.

\section{POSTURAL SYNERGIES}

In a recent series of studies, the described approach was used to analyze multi-muscle synergies involved in stabilizing vertical posture.
The studies involve an experimental identification of EVs (muscle modes or M-modes) based on indices of changes in the background EMG activity in anticipation of a self-triggered postural perturbation and at tite initiation of a voluntary whole-body sway (Krishnamoorthy et al., 2003a,b. 2004). Principal component analysis over repetitions of these tasks was used to identify stable muscle grouping within each subject. Note that we view such stable muscle groupings not as synergies (cf. Bizzi et al., 2002. Tresch et al., 1999) but rather as sets of EVs that can be manipulated by the controller to form task-specific synergies (Fig. 2).

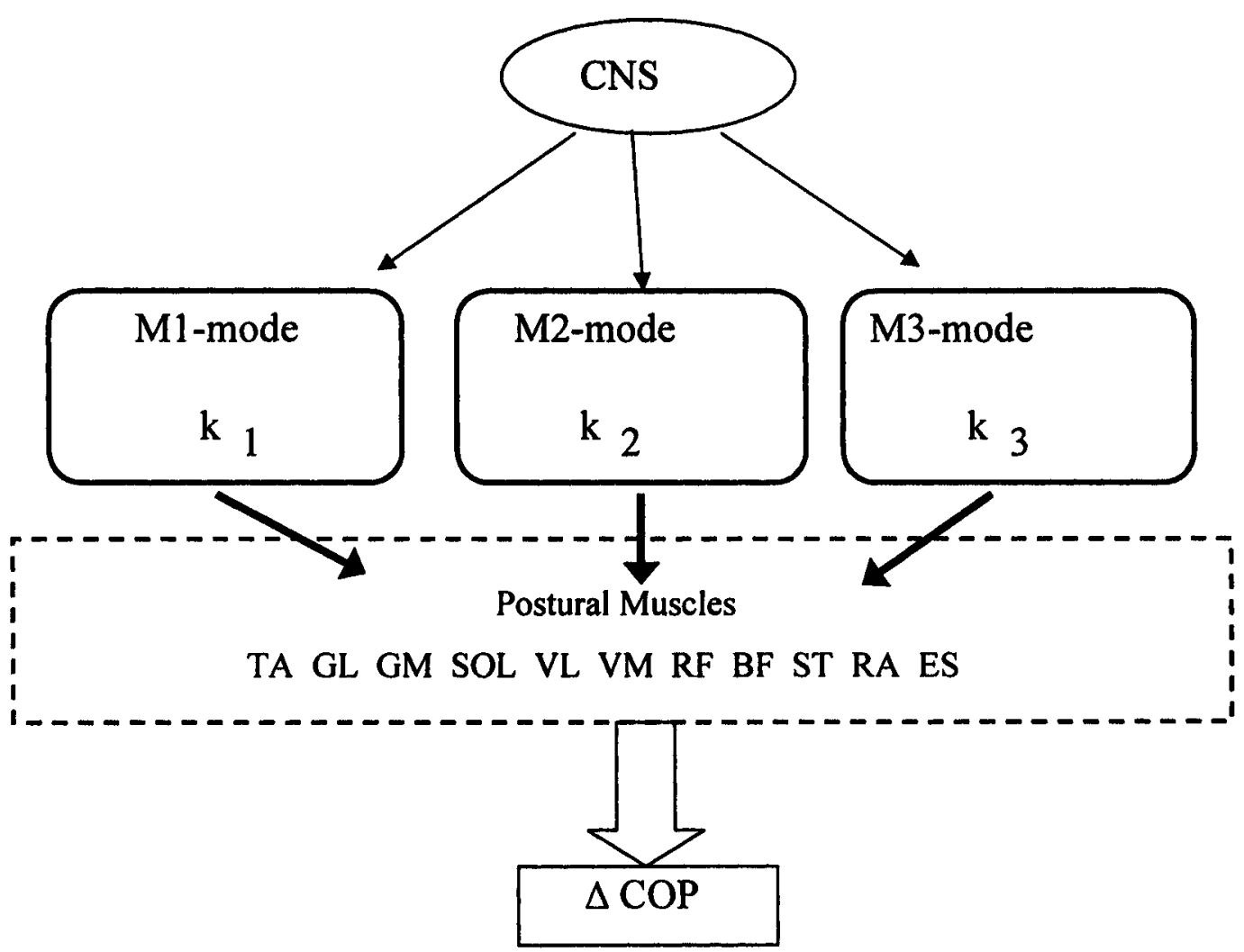

Fig. 2: An illustration of controlling shifts of the center of pressure ( $\triangle C O P$ ) with a set of muscle modes (M-modes). Each M-mode leads to parallel changes in the activity of a subset of postural muscles. The controller selects the magnitudes of the modes (reflected in coefficients $k$ ). 
Further, multiple regression analysis was used to compute a $\mathbf{J}$ matrix relating small changes in magnitudes of M-modes to shifts of the COP. Hence, these studies assumed that COP location is an important performance variable that can be stabilized by co-varied changes in the magnitudes of the M-modes (see Winter et al., 1996). The experiments have shown, in particular, that the same set of three M-modes could be used to form two different synergies that stabilized COP shifts forward and backwards.

Another recent study has provided evidence for error compensation among the joint oscillations that stabilize the horizontal position of the center of mass when standing quietly on a narrow base of support (Krishnamoorthy et al., 2005). A significant increase in overall joint variance was seen when subjects were asked to close their eyes. Most of this variance increase could be considered to reflect the error compensation feature of the postural synergy being channeled into the UCM. That is, when comparing the no-vision to the vision condition, only the variance component within the UCM ( $\mathrm{V}_{\mathrm{UCM}}$ ) increased significantly, whereas $\mathrm{V}_{\mathrm{ORT}}$ was not changed significantly.

\section{Changes in synergies with practice}

The UCM hypothesis and associated method of analysis allow quantifying changes in multielement synergies with practice. Several studies quantified changes in synergy indices with practice of such various tasks as two-arm pointing, multifinger force production, and Frisbee throwing (Domkin et al., 2003. Latash et al., 2003. Kang et al., 2004. Yang and Scholz, 2005). Taken together, the studies suggest two stages in the emergence and modification of synergies with practice (Fig. 3).
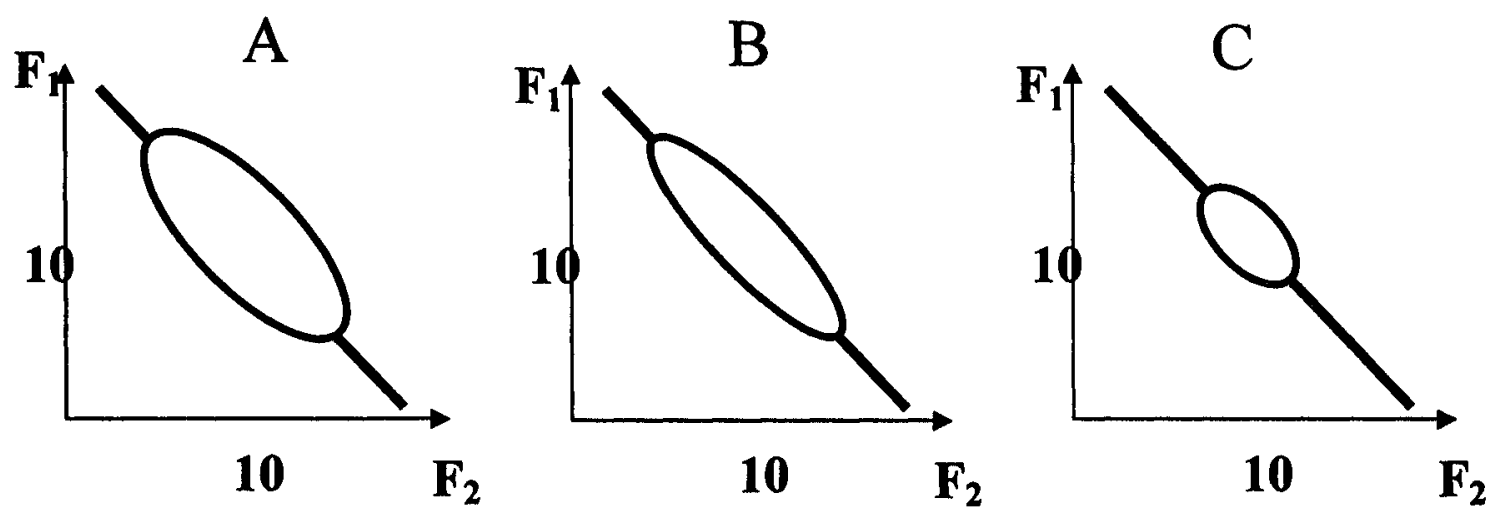

Fig. 3: An illustration of two stages of effects of practice on motor synergies. The ellipses illustrate data distributions for a set of trials to reach a certain summed output of two effectors $\left(F_{1}+F_{2}=20\right)$. The slanted lines correspond to the UCM for this task (see Fig. 1). During the first stage (from panel A to panel B), there is a decrease in the amount of variance that affects an important performance variable (orthogonal to the UCM, $V_{O R T}$ ) without a comparable decrease in the amount of variance that does not (parallel to the UCM, $V_{U C M}$ ). During the second stage (from panel $B$ to panel $C$ ), the situation reverses: There is a drop in $V_{U C M}$ without a comparable drop in $\mathrm{V}_{\text {ORT }}$. 
At the first stage, motor synergies emerge and become stronger in a sense that the "bad" component $\left(\mathrm{V}_{\mathrm{ORT}}\right)$ of the total variance drops at a faster rate such that the relative amount of "good" variance $\left(V_{U C M}\right)$ increases with practice (compare panels A and B in Fig. 3. Latash et al., 2003a. Kang et al., 2004).

During the second stage, the situation reverses: $\mathrm{V}_{\text {UCM }}$ shows a quicker decrease (compare panels $B$ and $\mathrm{C}$ in Fig. 3. Domkin et al., 2002. Latash et al., 2003), which looks counter-intuitive because this variance component does not affect important performance variables. Several interpretations have been suggested to account for the unexpected change in the two variance components during the second stage of practice effects. In particular, Domkin et al. (2002) suggested that the counterintuitive decrease in $\mathrm{V}_{\mathrm{UCM}}$ could be related to the formation of the more stereotypical families of trajectories that assure smooth transitions between successive UCMs. Alternatively, these effects could be related to an optimization of the performance with respect to factors other than the explicit performance variable, for example to minimization of the effects of transcranial magnetic stimulation as in the study of Latash and co-authors (2003).

\section{Atypical synergies in Down syndrome}

In one study (Scholz et al., 2003), the framework of the UCM hypothesis was applied to the analysis of the multi-finger production of an accurate slow ramp profile of the total force while persons with Down syndrome (DS), aged 14 to 42 , pressed on force sensors with all four fingers of the dominant hand (Latash et al., 2002b). Prior to practice, persons with DS showed predominantly positive covariation among individual finger forces (and modes) that destabilized the total force. Such patterns can be seen in typical persons only during the first few hundred of ms after the trial initiation and are quickly replaced by more adequate, negative finger force covariation (Shim et al., 2003). Persons with DS showed such patterns over the whole time of the ramp force production. The patterns of finger force covariation stabilized the total pronation/supination moment produced by all the fingers of persons with DS.

After two days of practice, persons with DS showed improved covariation of force modes to individual fingers, which stabilized the total force profile without a concomitant deterioration in the moment stabilization. This result carries two optimistic messages. First, persons with DS have ample room for improvement of multi-finger (and possibly other multi-element) synergies. Second, the UCM method is sensitive to changes in motor synergies that happen over a relatively short time, a few days.

The effects of practice on motor performance in multi-element, redundant systems have been traditionally viewed as a staged process that involves freezing and releasing degrees-of-freedom (Bernstein, 1967. Vereijken et al., 1992). The UCM-approach views the process of motor learning as leading to adjustments in an interaction of neural signals sent to all effectors. These adjustments are not expected to lead to involvement of new control variables into or purging some of the pre-existent control variables out of the interaction. The principal of motor abundance (Gelfand and Latash 1998) suggests that the excess degrees-of-freedom available to the motor system constitutes a 'blessing' rather than a 'curse', and greater exploitation of the available motor abundance should result in improved performance. The fact that persons with DS were able to explore and improve finger interaction over a relatively brief training period, and that the UCM analysis was able to reveal these changes suggest that this analysis will likely be sensitive to changes in postural synergies that occur during natural development as well as to changes that occur with practice. 


\section{IMPLICATIONS FOR POSTURAL DEVELOPMENT}

As no developmental study has been performed using the described approach to motor synergies, one can only speculate about possible changes that happen with postural synergies during typical and atypical development. We apologize for the inability to offer approaches that could distinguish between such important factors as genetics, maturation, and learning. Our attempts to apply the UCM approach to motor actions by atypically developing persons (Scholz et al., 2003) produced promising results and suggested that the approach can be used for studies of both typical and atypical development.

According to one of the dominant views, motor development proceeds as a continuous dialogue between the nervous system, the body, and the environment (Thelen \& Spencer, 1998). This view leads one to expect significant effects of naturally occurring exploratory actions by infants, as well as of specialized practice on the process of motor development, including the development of postural mechanisms. In particular, training has been shown to increase the probability of activation of functionally appropriate muscles (Sveistrup \& Woollacott, 1997). The importance of training has been emphasized in studies of both typically and atypically developing children, including those with Developmental Coordination Disorder (DCD) and with Down syndrome (Ulrich et al., 2001. Johnston et al., 2002. Adolph et al., 2003). Several components of postural multi-muscle synergies can be considered as separate potential sources of developmental changes.

First, the composition of stable muscle groupings (M-modes) used as EVs can be elaborated, changed, and become more stable across tasks and conditions. It has been shown that with increasing age, the variation in muscle activation patterns decreases (Hadders-Algra et al., 1996, 1997). This apparently more stereotypical pattern might reflect the emergence of stable M-modes. Studies of
Woollacott and colleagues (Sveistrup \& Woollacott, 1996. Woollacott \& Burtner, 1996) have shown a progression of postural responses elicited by platform perturbations during stance from activity in single or paired muscles. This progression typically starts in the ankle muscles, followed by the activation of larger postural muscle groups involving muscles crossing the more proximal joints in what the authors call "functional synergies". We would call these muscle modes. With respect to the control of sitting posture, qualitatively similar observations have been reported on the emergence and preferred use of the paraspinal-hamstring and paraspinal-quadriceps "synergies" (Harbourne et al., 1993).

Second, central mechanisms of M-mode covariation can emerge and be refined based on typical postural tasks as well as on the individual anatomy. Note that the relatively quick anatomical changes that occur in infants and toddlers can require continuous adjustments of both M-modes and their covariation to ensure stable postural control. In particular, postural adjustments have been shown to change with age, suggesting the development of control mechanisms reflecting the changing intersegmental mechanics (Haas et al., 1986). Apparently, the processes of creation of M-mode synergies can differ, depending on the complexity of the task. For example, an ability to adapt postural responses to external conditions emerges prior to the age of 1 year (Woollacott et al., 1998), whereas an ability to react efficiently to perturbations applied during gait initiation emerges only by 4 to 5 years (Wollacott \& Assaiante, 2002).

Third, postural synergies have to be triggered either by external signals, i.e. by postural perturbations, or internally, i.e. as in anticipatory postural adjustments (APAs) in preparation for an action that has destabilizing effects on the posture. APAs, which are generated prior to the prime mover activation, produce forces and torques acting against those expected from the planned 
action (reviewed in Massion, 1992). Apparent developmental changes in postural synergies might reflect the development of methods of triggering postural synergies. These changes can form, in particular, the basis for two transitions in postural development, described at 6 to 8 and at 12 to 15 months, the latter corresponding to the emergence of APAs (Van der Fits et al., 1999). Note, however, that later studies have suggested a much later emergence of APAs, by 2 to 11 years of age (van der Heide et al., 2003).

We think that the framework of the UCM hypothesis and its associated toolbox are potentially powerful methods for the analysis of changes in motor synergies induced by practice, natural aging, neurological disorder, atypical development, and typical development. In particular, these methods allow considering different potential contributors to the typical picture of postural development and provide a basis for their quantitative analysis. An ability to ask new questions is always a very exciting opportunity in scientific research.

\section{REFERENCES}

Adolph KE, Vereijken B, Shrout PE. 2003. What changes in infant walking and why. Child Dev 74: 475-497.

Alexandrov A, Frolov A, Massion J. 1998. Axial synergies during human upper trunk bending. Exp Brain Res 118:210-220.

Allum JH, Honegger F. 1993. Synergies and strategies underlying normal and vestibulary deficient control of balance: implication for neuro-prosthetic control. Prog Brain Res 97: 331-348.

Allum JH, Honegger F, Pfaltz CR. 1989. The role of stretch and vestibule-spinal reflexes in the generation of human equilibrating reactions. Prog Brain Res 80: 399-409.

Babinski N. 1899. De l'asynergie cerebelleuse. Revue Neurologique 7: 806-816.

Bernstein NA. 1947. On the Construction of Movements. Moscow: Medgiz (In Russian).
Bernstein NA. 1967. The Co-ordination and Regulation of Movements. Oxford, UK: Pergamon Press.

Bizzi E, D'Vella A, Saltiel P, Tresch M. 2002. Modular organization of spinal motor systems. Neuroscientist 8: 437-442.

Crenna P, Frigo C, Massion J, Pedotti. 1987. Forward and backward axial synergies in man. Exp Brain Res 65: 538-548

Danion F, Schöner G, Latash ML, Li S, Scholz JP, Zatsiorsky VM. 2003. A force mode hypothesis for finger interaction during multi-finger force production tasks. Biol Cybern 88: 91-98.

d'Avilla A, Saltiel P, Bizzi E. 2003. Combinations of muscle synergies in the construction of a natural motor behavior. Nat Neurosci 6: 300-308.

Domkin D, Laczko J, Jaric S, Johansson H, Latash ML. 2002. Structure of joint variability in bimanual pointing tasks. Exp Brain Res 143: 11-23.

Gelfand IM, Latash ML. 1998. On the problem of adequate language in movement science. Motor Control 2: 306-313.

Gelfand IM, Latash ML. 2002. On the problem of adequate language in biology. In: Latash $\mathrm{ML}$, ed, Progress in Motor Control. Vol. 2: StructureFunction Relations in Voluntary Movement. Urbana, Illinois, USA: Human Kinetics; 209-228.

Gelfand IM, Tsetlin ML. 1966. On mathematical modeling of the mechanisms of the central nervous system. In: Gelfand IM, Gurfinkel VS, Fomin SV, Tsetlin ML, eds, Models of the Structural-Functional Organization of Certain Biological Systems. Moscow: Nauka; 9-26. (in Russian, a translation is available in 1971 edition, Cambridge, Massachusetts, USA: MIT Press.)

Haas G, Diener HC, Bacher M, Dichgans J. 1986. Development of postural control in children: short-, medium-, and long latency EMG responses of leg muscles after perturbation of stance. Exp Brain Res 64:127-132.

Hadders-Algra M, Brogren E, Forssberg H. 1996. Ontogeny of postural adjustments during sitting in infancy: variation, selection and modulation. $J$ Physiol 493: 273-288.

Hadders-Algra M, Brogren E, Forssberg H. 1997. Nature and nurture in the development of postural control in human infants. Acta Paediatr Suppl 422: 48-53.

Harbourne RT, Giuliani C, Neela JM. 1993. A kinematic and electromyographic analysis of the development of sitting posture in infants. Dev Psychobiol 26: 51-64. 
Hughlings Jackson J. 1889. On the comparative study of diseases of the nervous system. Br Med J 355362.

Johnston LM, Burns YR, Brauer SG, Richardson CA. 2002. Differences in postural control and movement performance during goal directed reaching in children with developmental coordination disorder. Hum Move Sci 21: 583-601.

Kang N, Shinohara M, Zatsiorsky VM, Latash ML. 2004. Learning multi-finger synergies: An uncontrolled manifold analysis. Exp Brain Res 157: 336-350.

Krishnamoorthy V, Goodman SR, Latash ML, Zatsiorsky VM. 2003a. Muscle synergies during shifts of the center of pressure by standing persons: Identification of muscle modes. Biol Cybern 89: 152-161.

Krishnamoorthy V, Latash ML, Scholz JP, Zatsiorsky VM. 2003b. Muscle synergies during shifts of the center of pressure by standing persons. Exp Brain Res 152: 281-292.

Krishnamoorthy V, Latash ML, Scholz JP, Zatsiorsky VM. 2004. Muscle modes during shifts of the center of pressure by standing persons: Effects of instability and additional support. Exp Brain Res 157: 18-31.

Krishnamoorthy V, Yang J-F, Scholz JP. 2005. Joint coordination during quiet stance: Effects of vision. Exp Brain Res Apr 20; Epub PMID: 15841397.

Latash ML. 1996. How does our brain make its choices? In: Latash ML, Turvey MT, eds, Dexterity and Its Development. Mahwah, New Jersey, USA: Erlbaum Publ; 277-304.

Latash ML, Li ZM, Zatsiorsky VM. 1998. A principle of error compensation studied within a task of force production by a redundant set of fingers. Exp Brain Res 122: 131-138

Latash ML, Scholz JF, Danion F, Schöner G. 2001. Structure of motor variability in marginally redundant multi-finger force production tasks. Exp Brain Res 141: 153-165.

Latash ML, Scholz JP, Schöner G. 2002a. Motor control strategies revealed in the structure of motor variability. Exerc Sport Sci Rev 30: 26-31.

Latash ML, Kang N, Patterson D. 2002b. Finger coordination in persons with Down syndrome: atypical patterns of coordination and the effects of practice. Exp Brain Res 146: 345-355.

Latash ML, Scholz JF, Danion F, Schöner G. 2002c. Finger coordination during discrete and oscillatory force production tasks. Exp Brain Res 146: 412-432.

Latash ML, Yarrow K, Rothwell JC. 2003a. Changes in finger coordination and responses to single pulse TMS of motor cortex during practice of a multi-finger force production task Exp Brain Res 151: 60-71.

Latash ML, Danion F, Scholz JF, Schöner G. 2003b. Coordination of multi-element motor systems based on motor abundance. In: Latash ML, Levin MF, eds, Progress in Motor Control vol.3: Effects of Age, Disorder, and Rehabilitation. Urbana, Illinois, USA: Human Kinetics; 97-124.

Massion J. 1992. Movement, posture and equilibrium: Interaction and coordination. Prog Neurobiol 38: 35-56.

Nashner LM, Shupert CL, Horak FB, Black FO. 1989. Organization of posture controls: an analysis of sensory and mechanical constraints. Prog Brain Res 80: 411-418.

Newell KM, Carlton LG, Hancock PA. 1984. Kinetic analysis of response variability. Psychol Bull 96: 133-151

Newell KM, Carlton LG. 1988. Force variability in isometric responses. J Exp Psychol Hum Percept Perform 14: 37-44.

Newell KM, Corcos DM, eds. 1993. Variability and Motor Control. Urbana, Illinois, USA: Human Kinetics.

Scholz JP, Danion F, Latash ML, Schöner G. 2002. Understanding finger coordination through analysis of the structure of force variability. Biol Cybern 86: 29-39.

Scholz JP, Kang N, Patterson D, Latash ML. 2003. Uncontrolled manifold analysis of single trials during multi-finger force production by persons with and without Down syndrome. Exp Brain Res 153: 45-58.

Schöner G. 1995. Recent developments and problems in human movement science and their conceptual implications. Ecol Psychol 7: 291-314

Shim JK, Latash ML, Zatsiorsky VM. 2003. The central nervous system needs time to organize task-specific covariation of finger forces. Neurosci Lett 353: 72-74.

Shinohara M, Li S, Kang N, Zatsiorsky VM, Latash ML. 2003. Effects of age and gender on finger coordination in maximal contractions and submaximal force matching tasks. J Appl Physiol 94: 259-270.

Slijper H, Latash ML. 2000. The effects of instability 
and additional hand support on anticipatory postural adjustments in leg, trunk, and arm muscles during standing. Exp Brain Res 135: 81-93.

Sveistrup H, Woollacott MH. 1996. Longitudinal development of the automatic postural response in infants. J Mot Behav 28:58-70.

Sveistrup H, Woollacott MH. 1997. Practice modifies the developing automatic postural response. Exp Brain Res 114: 33-43.

Thelen E, Spencer JP. 1998. Postural control during reaching in young infants: 'a dynamic systems approach. Neurosci Biobehav Rev 22: 507-514.

Tresch MC, Saltiel P, Bizzi E. 1999. The construction of movement by the spinal cord. Nat Neurosci 2 : 162-167.

Turvey MT. 1990. Coordination. Am Psychol 45: 938-953.

Ulrich DA, Ulrich BD, Angulo-Kinzler RM, Yun J. 2001. Treadmill training of infants with Down syndrome: evidence-based developmental outcomes. Pediatrics 108: E84.

Van der Fits IB, Otten E, Klip AW, Van Eykern LA, Hadders-Algra M. 1999. The development of postural adjustments during reaching in 6- to 18month-old infants. Evidence for two transitions. Exp Brain Res 126: 517-528.

Van der Heide JC, Otten B, van Eykern LA, Hadders-Algra M. 2003. Development of postural adjustments during reaching in sitting children.
Exp Brain Res 151: 32-45.

Winter DA, Prince F, Frank JS, Powell C, Zabjek KF. 1996. Unified theory regarding $\mathrm{A} / \mathrm{P}$ and $\mathrm{M} / \mathrm{L}$ balance in quiet stance. J Neurophysiol 75: 2334-2343.

Woollacott M, Assaiante C. 2002. Developmental changes in compensatory responses to unexpected resistance of leg lift during gait initiation. Exp Brain Res 144: 385-396.

Woollacott MH, Burtner P. 1996. Neural and musculoskeletal contributions to the development of stance balance control in typical children and in children with cerebral palsy. Acta Paediatr Suppl 416: 58-62.

Woollacott MH, Burtner P, Jensen J, Jasiewicz J, Roncesvalles N, Sveistrup H. 1998. Development of postural responses during standing in healthy children and children with spastic diplegia. Neurosci Biobehav Rev 22: 583-589.

Yang J-F, Scholz JP. 2005. Learning a throwing task is associated with differential changes in the use of motor abundance. Exp Brain Res Jan 19; Epub PMID: 15657698.

Zatsiorsky VM, Li Z-M, Latash ML. 1998. Coordinated force production in multi-finger tasks. Finger interaction and neural network modeling. Biol Cybern 79: 139-150.

Zatsiorsky VM, Li Z-M, Latash ML. 2000. Enslaving effects in multi-finger force production. Exp Brain Res 131: 187-195. 

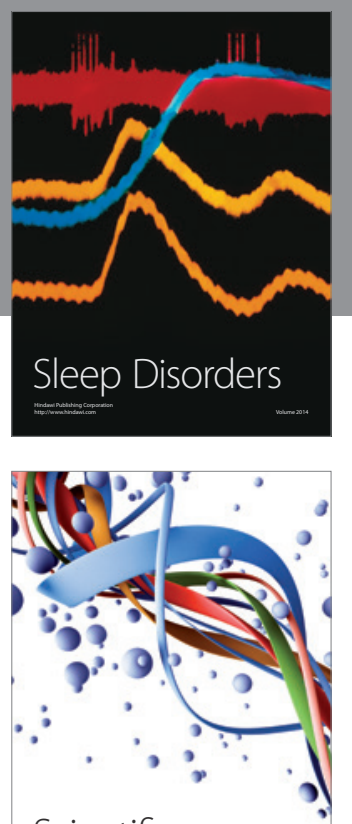

Scientifica
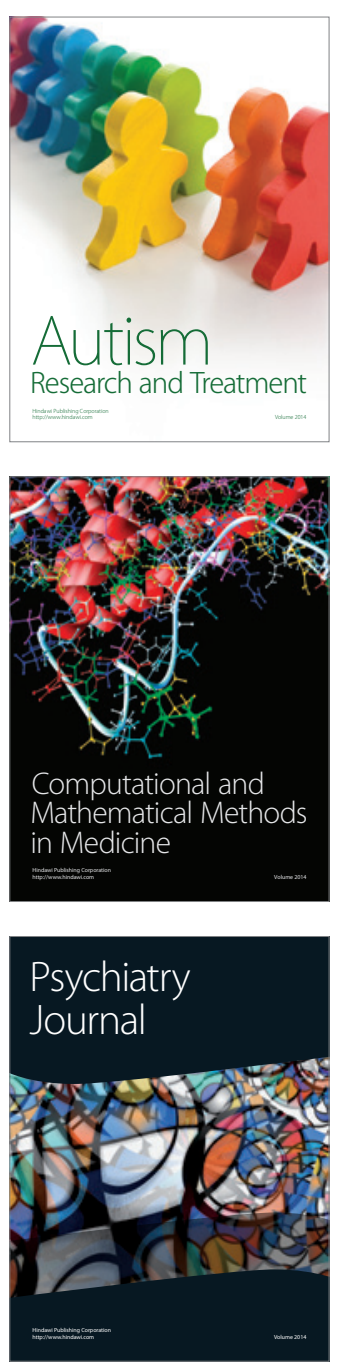
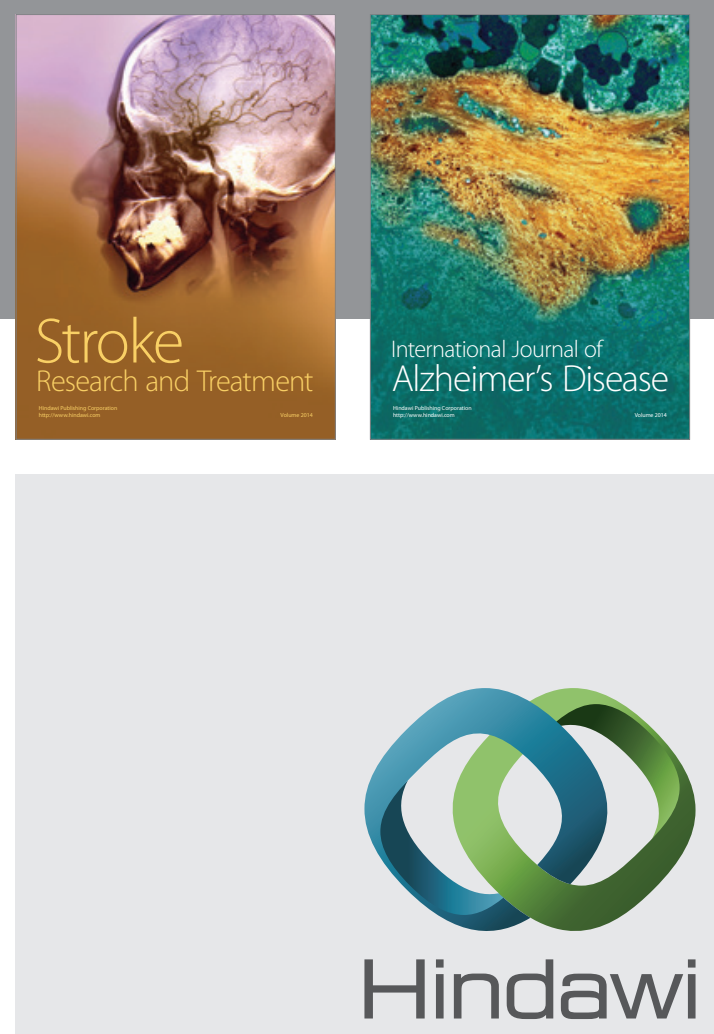

Submit your manuscripts at

http://www.hindawi.com
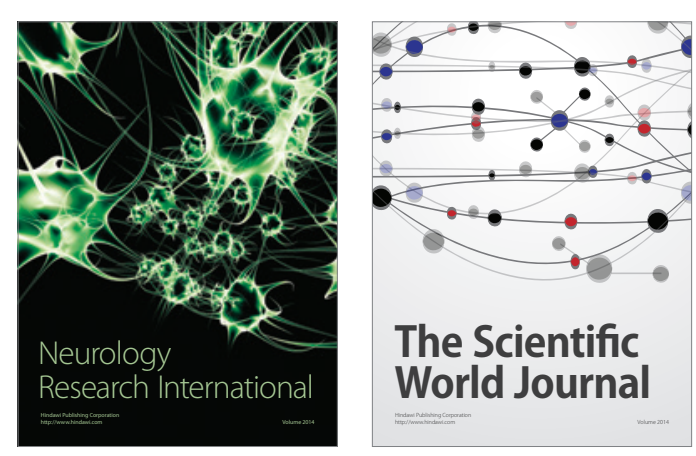

The Scientific World Journal

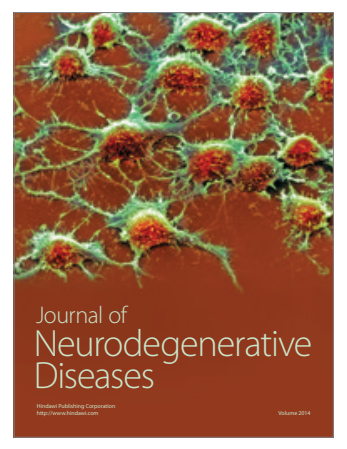

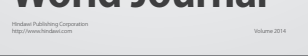

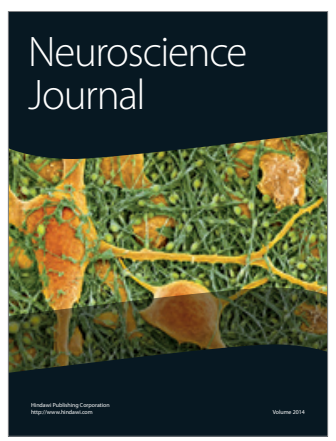

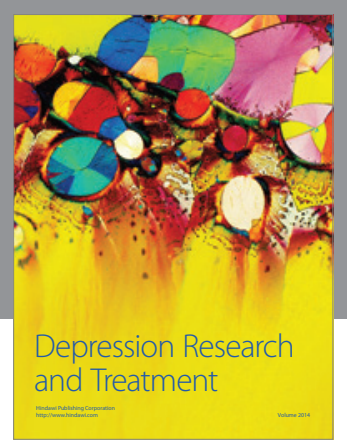
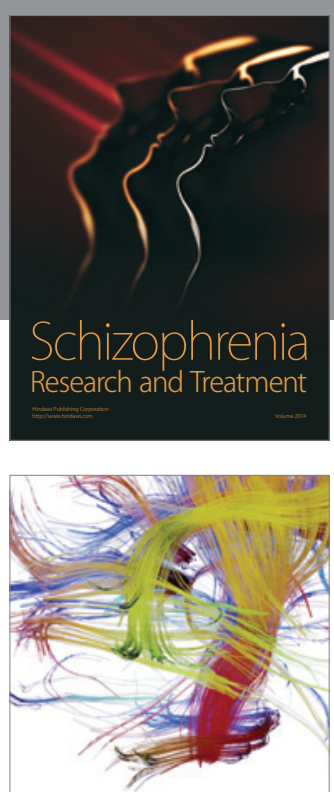

Brain Science

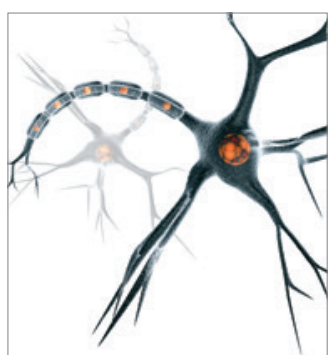

Neural Plasticity
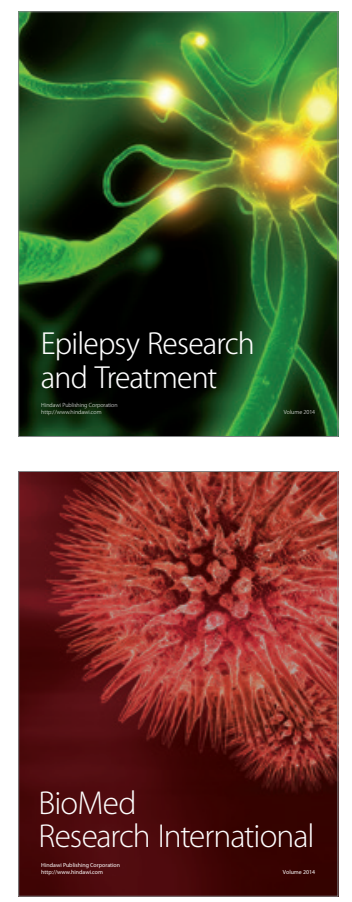

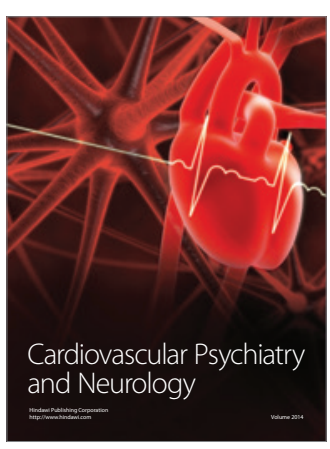

Parkinson's

Disease
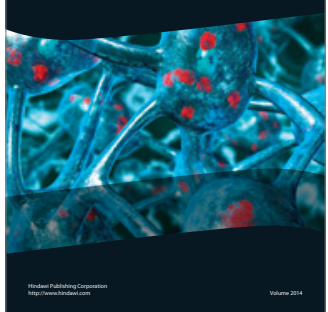